\title{
Finite Element Modeling of Gas and Temperature Distributions during Catalytic Reactions in an Environmental Transmission Electron Microscope
}

\author{
Jayse T. Langdon ${ }^{1}$, Joshua L. Vincent ${ }^{1 *}$, and Peter A. Crozier ${ }^{1}$ \\ 1. School for the Engineering of Matter, Transport, and Energy, Arizona State University, Tempe, Arizona \\ 85287-6106 \\ *Corresponding author: Joshua.Vincent@asu.edu
}

The application of environmental TEM (ETEM) to heterogeneous catalysts has led to many impressive reports on phase transformation pathways and structure-activity relationships [1]. Recent developments in specimen preparation techniques allow for measurable catalytic conversions to be generated in the ETEM, enabling operando correlations of structure with activity $[2,3]$. In brief, catalyst is dispersed on a porous glass fiber pellet and an inert metal grid, which are loaded into a heating holder. The large mass of catalyst on the pellet generates detectable conversions, while catalyst on the grid is imaged. The composition of product gases can be monitored with electron energy-loss spectroscopy (EELS). The operando pellet reactor geometry differs from the reactor architectures employed in reaction engineering. To determine kinetic parameters, it is necessary to establish a reactor model for the system. Here, we establish a finite element model of the operando pellet reactor and determine the temperature and gas profiles during catalysis. The model is applied to a $\mathrm{SiO}_{2}$-supported $\mathrm{Ru}$ catalyst performing $\mathrm{CO}$ oxidation.

An FEI Titan ETEM and a Gatan 628 single-tilt Ta heater holder are modeled with the COMSOL® Multiphysics finite-element simulation software. The Computational Fluid Dynamics, Heat Transfer, and Chemical Reaction Engineering modules are used. Bulk fluid flow is modeled with the Navier-Stokes equation, while flow in the porous pellet is modeled by the Darcy-Brinkman equation. Multi-component diffusion is modeled with the Maxwell-Stefan equation. Heat transfer considerations include conduction, convection, and radiation. The catalytic reaction was modeled as irreversible and elementary, with an activation energy of $90 \mathrm{~kJ} / \mathrm{mol}$ [4]. The catalyst in the pellet was modeled with an egg-shell distribution. Experimentally, the mass of catalyst is not known exactly (typically it is on the order of $20 \mu \mathrm{g}$, while the mass of the pellet is $\sim 3000 \mu \mathrm{g}$ ), so in this work the forward frequency factor, $A$, has been adjusted until a match was achieved for experimental conversions measured at $340{ }^{\circ} \mathrm{C}$ (here, $A=7 * 10^{12} \mathrm{~s}^{-1}$ ). Material properties and thermo-physical parameters were taken from tabulated sources.

Views of the modeled environmental cell and operando pellet reactor geometry are shown in Figures 1(a) and 1(b), respectively. Gas flows into the ETEM cell from the inlet on the left, and it leaves through the pumping apertures in the pole pieces. The gas composition measured experimentally by EELS can be simulated with a line integral along the path labeled "EELS Line" in Figure 1(b). In Figure 2, the temperature distribution in the pellet and surrounding the furnace is shown for a set-point of $230{ }^{\circ} \mathrm{C}$, which corresponds to an EELS conversion measurement of $32 \%$. At this condition the temperature distribution is seen to be largely uniform in the pellet and furnace, rapidly decreasing outside this region to $25{ }^{\circ} \mathrm{C}$ at the water-cooled pole piece surfaces. The distribution of $\mathrm{CO}_{2}$ at the same condition is shown in Figure 3. The concentration of $\mathrm{CO}_{2}$ is highest in the pellet $(\sim 38 \%)$, which is expected since the catalyst is located there. Interestingly, the concentration decreases only slightly to $\sim 32 \%$ in the environmental cell around the operando pellet reactor. A comparison of simulated conversions with experimental measurements spanning a temperature range of $25-350{ }^{\circ} \mathrm{C}$ (not shown here) show good agreement. It is hoped that this model will become an accessible tool for other experimentalists to adapt to their systems of interest [5]. 


\section{References:}

[1] Crozier, P. A., and Hansen, T. W.; MRS Bulletin, 2015, 40, pp. 38-45.

[2] Chenna, S., and Crozier, P. A.; ACS Catalysis, 2012, 2(11), pp. 2395-2402.

[3] Miller, B. K., Barker, T. M., and Crozier, P. A.; Ultramicroscopy, 2015, 156, pp. 18-22.

[4] Miller, B. K., and Crozier, P. A.; Microscopy and Microanalysis, 2014, 20, pp. 815-824.

[5] We gratefully acknowledge the support of NSF grants CBET-1134464 and CBET-1604971, as well as ASU's John M. Cowley Center for High Resolution Electron Microscopy.
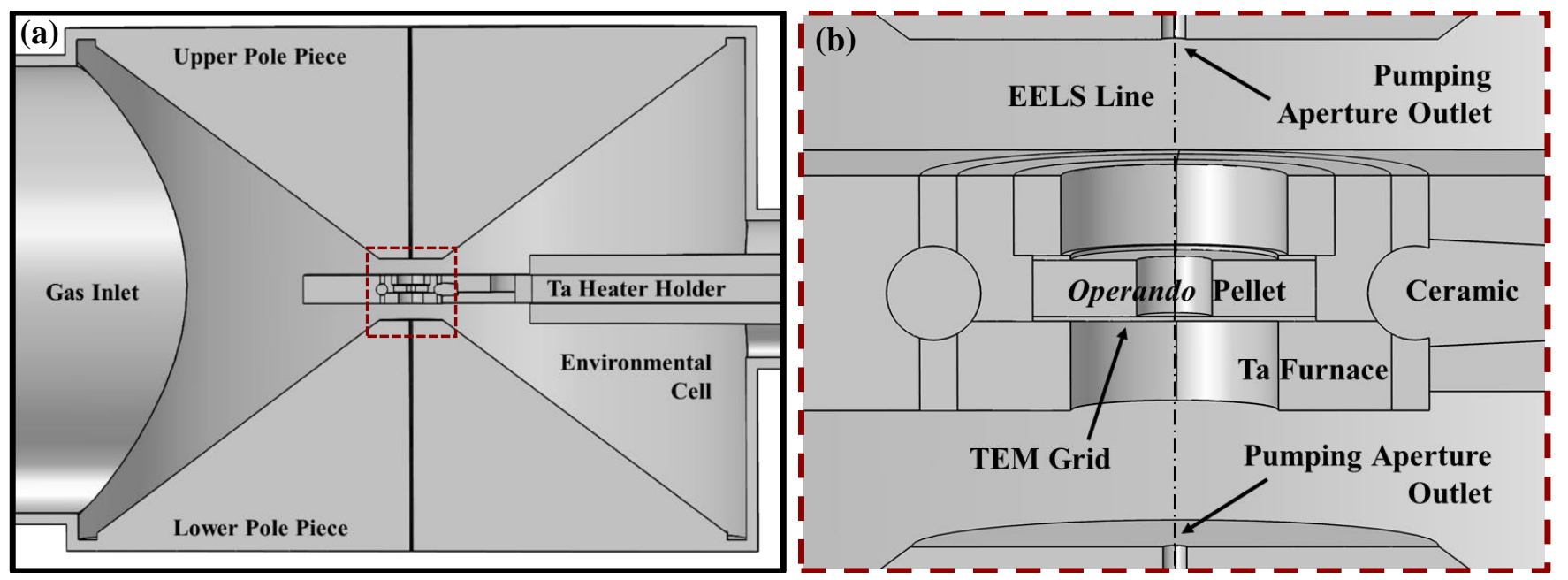

Figure 1. Environmental cell model geometry in full view (a). The region contained by the red dashed box contains the operando pellet reactor, which is shown in more detail in (b). Note that the model takes advantage of the mirror plane symmetry present in the environmental cell.

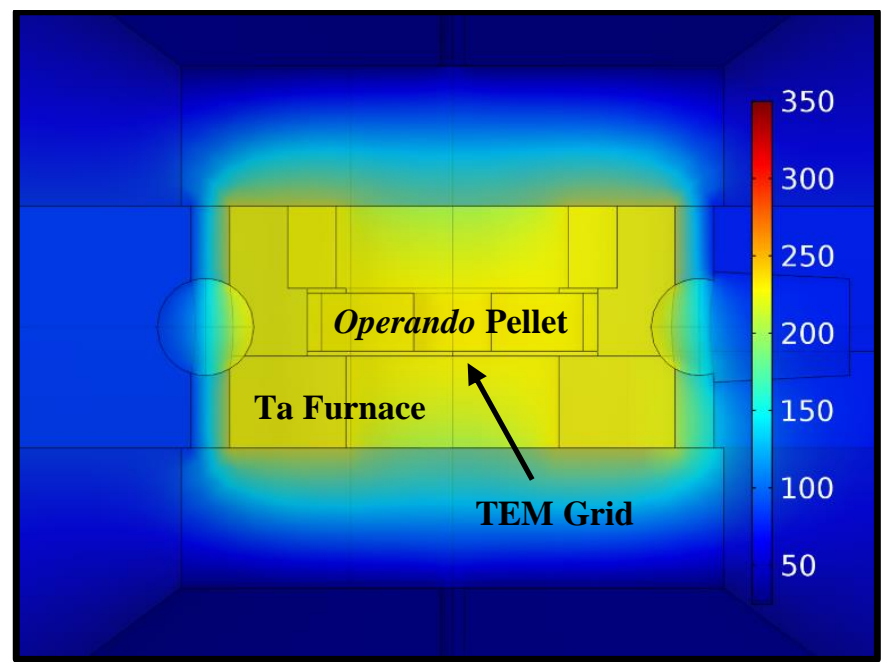

Figure 2. Temperature distribution $\left({ }^{\circ} \mathrm{C}\right)$ in operando catalyst pellet and around furnace for set-point of $230{ }^{\circ} \mathrm{C}$ in 2.2 Torr of a stoichiometric mixture of $\mathrm{CO}$ and $\mathrm{O}_{2}$. The simulated conversion measured with EELS at this condition is $32 \%$. It can be seen that the temperature distribution in the vicinity of the catalyst is largely uniform.

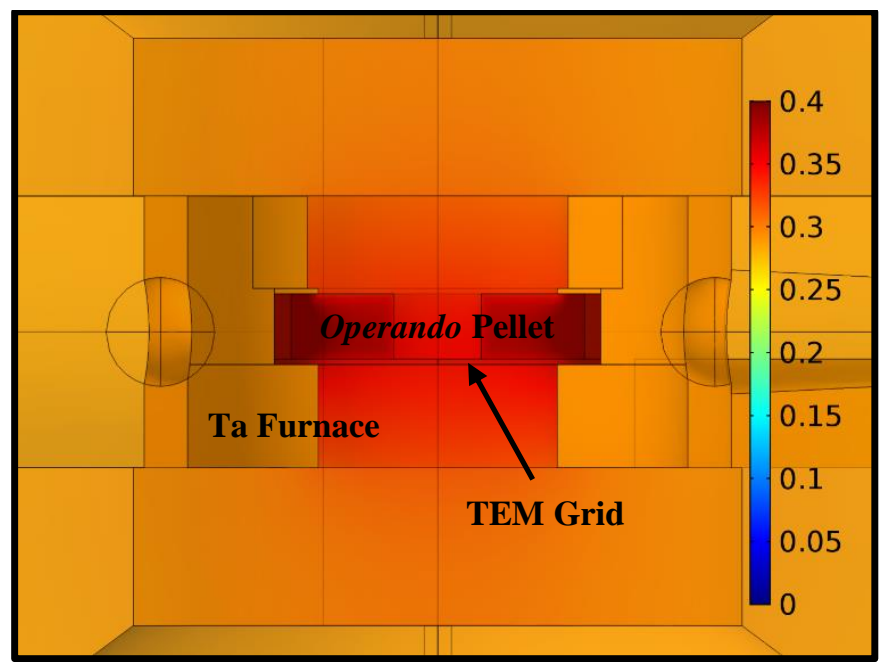

Figure 3. Mole fraction of $\mathrm{CO}_{2}$ in the operando pellet and near the holder at same the conditions simulated in Figure 2. The concentration of catalytically produced $\mathrm{CO}_{2}$ is highest in the operando pellet, as expected, and decreases only slightly $(\sim$ few $\%)$ in the cell surrounding the pellet at this condition. 